\title{
Mesure des efforts de défonçage et de la qualité finale en usinant en différents angles du fil : comparaison entre le pin Douglas et le pin d'Alep
}

\author{
Hamid AKNOUCHE ${ }^{1}$ \\ Giacomo GoLI ${ }^{2}$ \\ Rémy MARCHAL ${ }^{3}$ \\ Jakub SANDAK ${ }^{4}$ \\ Abdellatif ZERIZER ${ }^{1}$ \\ Jean-Claude ButAUd ${ }^{3}$ \\ ${ }^{1}$ Université M’Hamed Bougara \\ de Boumerdès \\ UR-MPE \\ Avenue de l'Indépendance \\ 35000 Boumerdès \\ Algérie \\ 2 Université de Florence \\ Deistaf \\ Piazzale delle Cascine \\ 50144 Florence \\ Italie \\ ${ }^{3}$ Centre arts et métiers \\ ParisTech de Cluny \\ Laboratoire bourguignon des \\ matériaux et procédés (LaBoMaP) \\ Rue Porte de Paris \\ 71250 Cluny \\ France \\ ${ }^{4}$ Conseil national de la \\ recherche/IVALSA \\ San Michele all'Adige \\ Via Biasi, 75 \\ 38010 - San Michele all'Adige (TN) \\ Italie
}

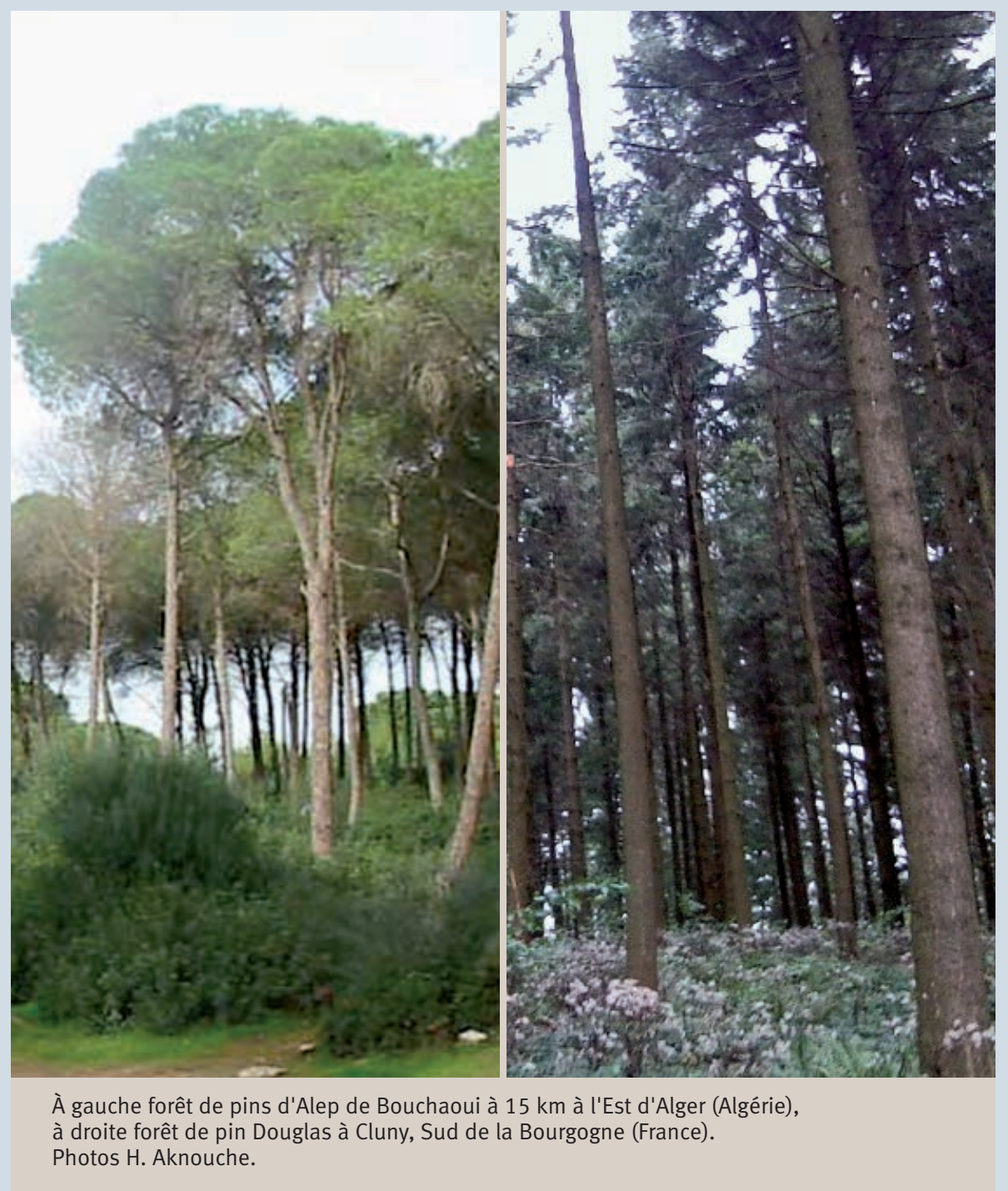




\section{RÉSUMÉ}

\section{MESURE DES EFFORTS DE DÉFONÇAGE ET DE LA QUALITÉ FINALE EN USINANT EN DIFFÉRENTS ANGLES DU FIL : COMPARAISON ENTRE LE PIN DOUGLAS ET LE PIN D’ALEP}

Le travail a pour but d'effectuer une comparaison entre un bois très commun et usiné en Europe comme le pin Douglas et une essence pas très utilisée comme le pin d'Alep de provenance algérienne. Pour cette comparaison, une nouvelle méthode a été mise au point afin d'évaluer les efforts de coupe et la qualité des surfaces usinées dans le processus du défonçage. Cette méthode a été expérimentée sur du pin d'Alep en coupe orthogonale allant de façon continue de $90^{\circ} / 0^{\circ}$ à $90^{\circ} / 90^{\circ}$ dans le sens du fil et contrefil afin de juger également de l'aptitude à l'usinage de ce bois par rapport à d'autres essences plus communément usinées. L'outil de coupe est une fraise circulaire comportant deux plaquettes en carbure. Elle est utilisée pour usiner le chant de disques de pin d'Alep prélevés dans une planche, ce qui induit une variation de l'angle du fil continue de $0^{\circ}$ à $360^{\circ}$. La mesure des forces de coupe a été faite avec l'emploi d'une table piézoélectrique Kistler 9257A à trois axes montée sur la table d'une défonceuse à commande numérique, couplée à un PC et une carte d'acquisition. Les données sont traitées à l'aide d'un logiciel Dasylab. Parallèlement, des tests de qualité des surfaces usinées avec un outil comportant une arête de coupe neuve sur une moitié et usée artificiellement sur l'autre ont été réalisés par une nouvelle méthode sans contact développée par l'équipe de recherche $\mathrm{Cnr}$ /lvalsa. Les résultats indiquent que les efforts de coupe sont un peu plus grands pour le pin Douglas que pour le pin d'Alep. En revanche, la qualité des surfaces obtenues est bien meilleure pour le pin d'Alep que pour le pin Douglas.

Mots-clés : pin d'Alep, pin Douglas, défonçage, orientation du fil, effort de coupe, rugosité, Algérie.

\section{ABSTRACT}

\section{MEASURING CUTTING EFFORT AND THE SURFACE QUALITY OBTAINED BY ROUTING ACROSS DIFFERENT WOOD GRAIN ANGLES: A COMPARISON BETWEEN DOUGLAS PINE AND ALEPPO PINE}

The aim of this study was to compare a timber species commonly machined in Europe, in this case Douglas pine, with a little used timber species, in this case Aleppo pine from Algeria. A new method was developed to assess the cutting effort and the quality of the machined surfaces. The method was tested with Aleppo pine using a wood router held perpendicular to the surface and cutting continuously from $90^{\circ} / 0^{\circ}$ to $90^{\circ} / 90^{\circ}$ with or against the grain, so that the machining properties of the wood could also be assessed in comparison with other more commonly used timber species. The router bit, a rotary cutter with two carbide inserts, was used to bevel the edges of Aleppo pine discs cut from a board, which required the cutter to move continuously from an angle to the wood grain of $0^{\circ}$ through to $360^{\circ}$. The cutting forces were measured using a 3-axis Kistler 9257A piezoelectric transducer fixed to the table of a digitally controlled router connected to a PC and a video capture board. The data were processed with Dasylab software. In parallel, the quality of the machined surfaces was tested with a tool where half of the cutting edge was new and the other half artificially blunted, using a new contact-free method developed by the CNR/ IVALSA research team. The results show that a little more cutting effort is required with Douglas pine than with Aleppo pine, and that the quality of the cut surfaces is far better with Aleppo pine than Douglas pine.

Keywords: Aleppo pine, Douglas pine, router, wood grain angle, cutting effort, evenness, Algeria.

\section{RESUMEN}

\section{MEDIDA DE LOS ESFUERZOS DE CAJEADO Y DE LA CALIDAD FINAL EN MECANIZADO CON DISTINTOS ÁNGULOS DE HILO: COMPARACIÓN ENTRE EL PINO DE OREGÓN Y EL PINO CARRASCO}

El objetivo de este trabajo consistía en comparar una madera muy común y habitualmente mecanizada en Europa como la del pino de Oregón con una especie no muy empleada como el pino carrasco de origen argelino. Para realizar dicha comparación se puso a punto un nuevo método con el fin de evaluar el esfuerzo cortante y la calidad de las superficies mecanizadas en el proceso de cajeado. Este método se experimentó en pino carrasco en corte ortogonal variando de forma continua de $90 / 0^{\circ}$ a $90^{\circ} / 90^{\circ}$, al hilo y a contrahílo, para examinar también la aptitud para el mecanizado de esta madera con relación a otras especies de mecanizado más frecuente. Como herramienta de corte se empleó una fresa circular con dos plaquitas de carburo que se utilizó para mecanizar cantos de discos de pino carrasco extraídos de una tabla, lo que provoca una variación continua del ángulo del hilo de $0^{\circ} \mathrm{a}$ $360^{\circ}$. La medida de las fuerzas de corte se realizó mediante una plataforma piezoeléctrica Kistler 9257A de tres ejes montada en la mesa de una rebajadora de control digital, acoplada a una PC y a una tarjeta de adquisición. Los datos se procesaron mediante el software DASYLab. En paralelo, y siguiendo un nuevo método sin contacto desarrollado por el equipo de investigaciones CNR/IVALSA, se efectuaron pruebas de calidad de las superficies mecanizadas con una herramienta equipada con un borde cortante nuevo en una mitad y artificialmente desgastado en la otra. Los resultados indican que el esfuerzo cortante es un poco mayor en el pino de Oregón que en el pino carrasco. Sin embargo, la calidad de las superficies obtenidas es mucho mejor en el pino carrasco que en el pino de Oregón.

Palabras clave: pino carrasco, pino de Oregón, cajeado, dirección del hilo, esfuerzo cortante, rugosidad, Argelia. 


\section{Introduction}

Le pin d'Alep est une essence méditerranéenne encore peu valorisée en bois d'œuvre alors qu'il est avéré que ses propriétés intrinsèques le mettent à un niveau proche de celui du pin maritime largement transformé, dans le massif landais français notamment (THIBAUT et al., 1992). Il convient ici de vérifier son aptitude à l'usinage par une analyse des efforts de coupe mis en jeu lors de sa transformation et de la qualité des surfaces obtenues. Celle-ci dépend de très nombreux facteurs dont l'un des plus importants est l'orientation du fil. Elle a une influence primordiale sur le comportement mécanique du bois en général.

L'acquisition des efforts de coupe nous renseigne sur le processus qui se développe pendant la formation de la surface (STEWART, 1969 ; Goli et al., 2009a, b ; WYETH et al., 2009), sur la qualité des surfaces usinées (STEWART, 1969 ; CYRA, TANAKA, 2000 ; Gol et al., 2001), et sur l'état d'usure des outils de coupe (Aguillera, Martin, 2001 ; AKNouche et al., 2009). Dans notre cas, l'étude est axée sur l'analyse des variations des efforts de coupe avec celles de l'angle du fil dans le cas du fraisage en avalant du pin d'Alep.

La force résultante agissant sur le bois peut être décomposée en une force parallèle (FP) et une force normale (FN) par rapport à la surface générée (figure 1). La magnitude et la direction des composantes parallèle et normale de la force résultante vont dépendre des divers facteurs propres à l'outil de coupe, aux conditions de travail ainsi qu'au matériau travaillé (KoCH et al., 1985 ; MARCHAL et al., 2009). La variation des forces en fonction des diverses variables de coupe a fait l'objet de plusieurs études. Ainsi, les efforts de coupe ont été étudiés en fonction de l'angle d'attaque (WoOdson, $\mathrm{KoCH}$, 1970 ; NeRI et al., 1999), de l'épaisseur du copeau (WOOdSON, KOCH, 1970 ; NeRI et al., 2000), de l'orientation des couteaux (STEWART, 1980), de l'usure des outils (STEWART, 1986 ; HERNÁNDEZ, RojAS, 2002), ainsi que de la masse volumique (NERI et al., 2000), de la teneur en humidité et de la température du bois (JIN, CAI, 1997).

Pour tenir compte de tous ces facteurs tout en réduisant le temps d'expérimentation, une nouvelle méthode a été mise au point et expérimentée. Cette méthode consiste à usiner un disque à partir d'une planche placée sur une plateforme dynamométrique, ce qui permet de mesurer les efforts de coupe pour tous les angles du fil, tout en générant toutes les qualités de surface pour différents angles du fil. Une fois la pièce usinée, la qualité finale est quantifiée à l'aide d'une caméra et d'un capteur laser balayant la surface à caractériser, en faisant tourner le disque à l'aide d'un moteur pas à pas et en exécutant le balayage dans la direction d'usinage et perpendiculairement à cette direction.

Le contexte de cette étude a été amorcé lors des travaux de thèse de AKNouche (2009) ${ }^{1}$.

\section{Matériels et méthodes}

\section{Défonçage du bois}

Les essais ont été conduits sur la défonceuse à commande numérique Scm Record1 du LaBoMaP à Cluny. L'outil à deux plaquettes carbure avait un diamètre de $80 \mathrm{~mm}$ (photo 1a) et tournait à 6000 tours par minute. Seule une plaquette travaillait, l'autre, usée, assurant seulement l'équilibre de l'outil.

Quelques essais préliminaires ont été réalisés en vue d'optimiser la procédure d'acquisition des efforts.

Pour s'affranchir de l'influence de la variabilité du bois, il a été utilisé des échantillons pris de façon aléatoire dans un lot d'avivés issus de 19 arbres de pin d'Alep de la région d'Aubagne (France). L'âge moyen de ces arbres était de 60 ans pour un diamètre moyen de $36 \mathrm{~cm}$ (32 à $40 \mathrm{~cm}$ ). Les échantillons sont constitués à $90 \%$ de duramen, l'aubier ayant été purgé en grande partie lors du sciage. La densité moyenne des éprouvettes est de $550 \mathrm{~kg} / \mathrm{m}^{3}$ à l'humidité de référence (12\%) (tableau I).

Les éprouvettes au nombre de cinq sont de forme carrée, de dimensions $240 \times 240 \times 35 \mathrm{~mm}$. Elles comportent deux perçages de diamètre $8 \mathrm{~mm}$ pour leur fixation sur la table piézoélectrique, elle-même fixée sur la table de la défonceuse (photo $1 \mathrm{~b}$ ).

La plaquette de l'outil est en carbure WC-Co, avec un angle d'attaque de $25^{\circ}$ et de bec de $55^{\circ}$. Les différents paramètres de coupe sont donnés dans le tableau II.

\section{${ }^{1}$ AKNOUCHE H., 2009. Étude de l'usure d'outils de coupe traités dans le domaine de l'usinage du bois. Thèse de doctorat, Université M'Hamed Bougara, Boumerdès, Algérie. \\ http://bu.umbb.dz/ressources-electroniq/theses-n/polymers-et-} composites/Aknouche-Hamid.pdf.

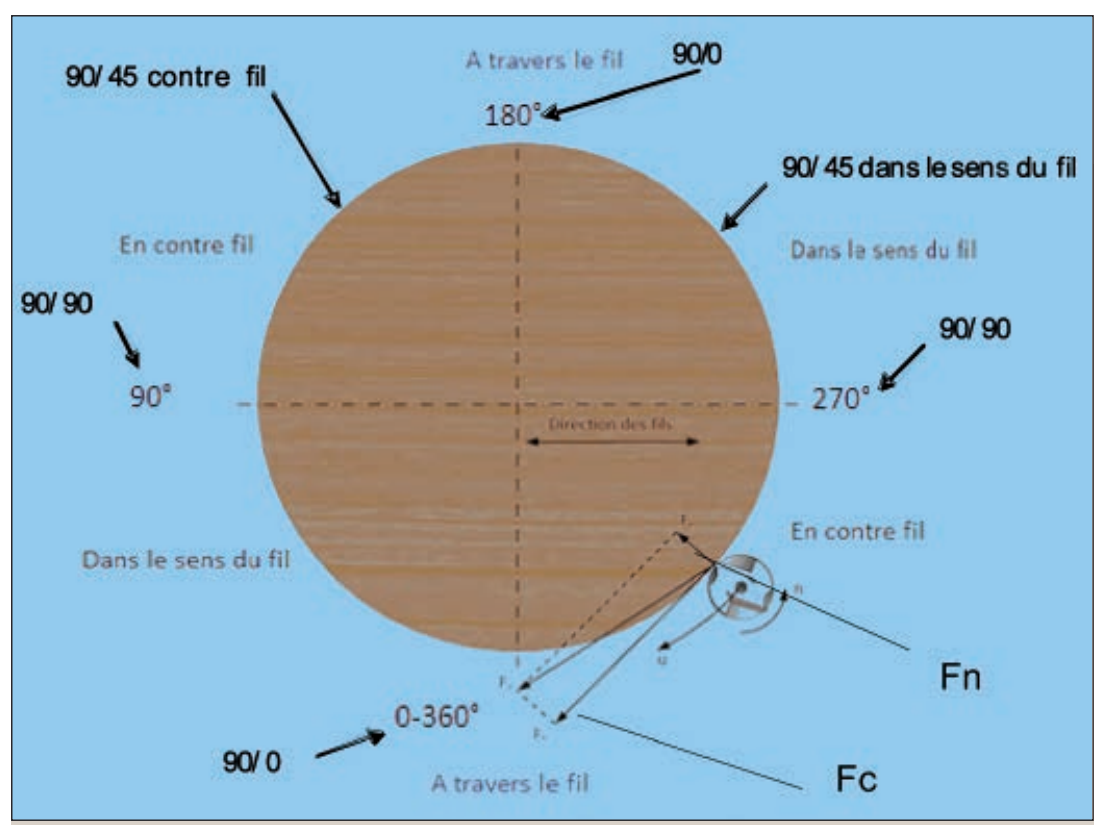

Figure 1.

Efforts agissant lors du défonçage. 


\section{Mesure et acquisition des efforts de coupe}

La mesure des efforts de coupe pendant le processus de défonçage est une opération compliquée par la haute fréquence et la sollicitation périodique qui excitent le système selon sa fréquence propre. Le système de mesure est une plateforme piézoélectrique dynamométrique à trois axes de marque Kistler 9257A, reliée à trois amplificateurs de charge. Les forces de coupe ont été mesurées pendant le défonçage suivant la direction longitudinale et radiale ( $X$ et $Y$ ), et la force de coupe résultante a été calculée. Les données acquises ont été analysées avec le logiciel Dasylab développé par la compagnie Norton.

L'analyse des forces s'est faite en incorporant des filtres passe-bas d'ordre 4 coupant à $500 \mathrm{~Hz}$ afin de diminuer le bruit et d'avoir des résultats comparables avec les données de la bibliographie (Goll et al., 2009b). La fréquence du filtre est supérieure à celle de la coupe $(100 \mathrm{~Hz})$, le filtrage ayant pour conséquences de gagner en qualité de lecture, mais les efforts de coupe peuvent être légèrement modifiés. Les conditions d'analyse sont données dans le tableau III.

\section{Image numérique de la surface}

L'essai a consisté à usiner le chant des éprouvettes initialement carrées pour les mettre au rond puis exécuter des cycles d'usinage circulaire avec une profondeur de passe de $2 \mathrm{~mm}$. Cela permet, tout en conservant la même profondeur de passe, de faire varier progressivement l'angle du fil en passant du mode $90^{\circ} / 90^{\circ}$ (MCKenzIE, 1962) (arête de l'outil et direction de coupe perpendiculaire à la fibre - c'est-à-dire coupe en bout) au mode $90^{\circ} / 0^{\circ}$ (coupe de type dressage), en usinant à contrefil comme dans le sens du fil (figure 1).
Pour pouvoir obtenir une photo de la surface, une caméra numérique a été utilisée. La caméra de type Pixelink PL-A782 avec 6,6 Mpixels équipée d'un objectif de type Optoengineering MC3-03X est fixée de la même façon que le laser dans la photo $2 \mathrm{a}$. La rotation du disque s'est faite avec un pas de $0,1^{\circ}$ et à chaque pas une photo de $8 \times 3000$ pixels était enregistrée. Enfin, les lignes ont été recomposées dans une seule image de $28800 \times 3000$ pixels.

Tableau II.

Paramètres de coupe.

Vitesse de rotation de l'outil : 6000 tours/min

Avance de l'outil : 7,5 m/min

Profondeur de passe : $2 \mathrm{~mm}$

Technique de fraisage : avalant, $90^{\circ} / 90^{\circ} 90^{\circ} / 0^{\circ}$ dans le sens du fil et contrefil. Nombre de couteaux sur la fraise : 1 
Tableau III.

Paramètres d'analyse des efforts de coupe.

Désignation

Fréquence de résonance de la plateforme

Fréquence de prélèvement

Temps d'un tour

Fréquence de coupe

Valeurs
$\begin{aligned} & 3,5 \mathrm{kHz} \\ & 10 \mathrm{kHz} \\ & 0,01 \mathrm{~s} \\ & 100 \mathrm{~Hz}\end{aligned}$

Valeurs

$500 \mathrm{~Hz}$

Quatrième

Basse fréquence

Trois axes, piézoélectrique

\section{Mesure de la rugosité}

Les défauts d'usinage en surface ont été mesurés avec un capteur laser de marque Keyence - type LK-G32, avec un diamètre du faisceau laser de $30 \mu \mathrm{m}$, un champ de mesure de $10 \mathrm{~mm}$, une précision de $\pm 0,05 \%(5 \mu \mathrm{m})$ et une répétabilité de 0,05 $\mu \mathrm{m}$. Les disques usinés ont été montés sur un axe tournant (photo $2 b$ ) et les surfaces ont été explorées par le laser en position fixe pour obtenir un profil de rugosité. Deux procédures d'exploration ont été adoptées pour tester l'efficacité des différentes méthodes et ainsi pouvoir fournir différents paramètres descriptifs des surfaces :

- profil longitudinal complet, le laser est en position fixe et la roue fait un tour complet ; le profil est mesuré sur la périphérie de la roue usinée, dans la direction d'usinage ; - profil transversal, la roue tourne d'une manière séquentielle d'un angle de $10^{\circ}$ et le profil est mesuré transversalement, sur la largeur de coupe.

La mesure du profil longitudinal complet a l'avantage d'être très rapidement effectuée (environ 1 minute) mais ce profil sur une seule ligne n'est pas nécessairement représentatif de la surface intégrale (notamment du fait de proportions bois de printemps / bois d'été pouvant être très variables). C'est pourquoi nous avons réalisé d'autres essais en mesurant des profils transversaux tous les $10^{\circ}$ (36 mesures) qui prennent des temps de mesure plus longs (5-6 minutes).

Les profils ont été analysés par le logiciel Gwyddion. Les trois profils ont été mis à l'échelle permettant une analyse correcte, l'erreur de forme (l'usinage n'était pas un cercle parfait mais plutôt une ellipse) a été filtrée de façon à laisser seulement les défauts d'usinage, le profil a été mis à plat et la valeur moyenne de déformation a été fixée à la valeur zéro. Enfin, les analyses statistiques ont été effectuées avec le logiciel R. Pour les 36 profils, une surface tridimensionnelle a été reconstruite et analysée avec les mêmes outils que les profils 2D.

Le disque de pin d'Alep a été usiné avec un outil neuf sur une moitié de son épaisseur et usé artificiellement sur l'autre moitié de façon à analyser l'influence de l'usure sur les états de surface.

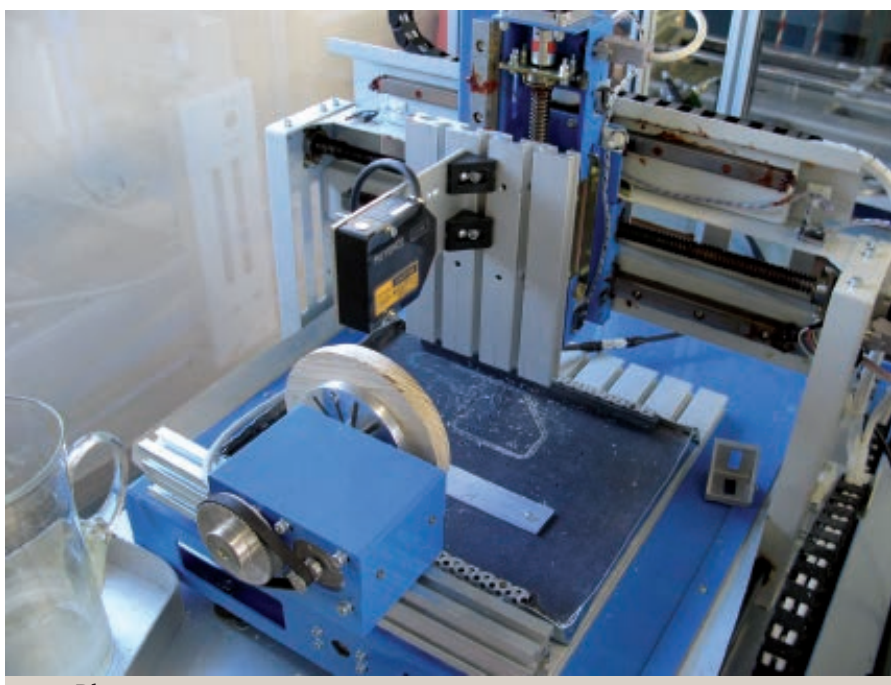

Photo 2a

Banc d'essai du test de la qualité de surface. Position du disque sur l'axe tournant.

Photo H. Aknouche.

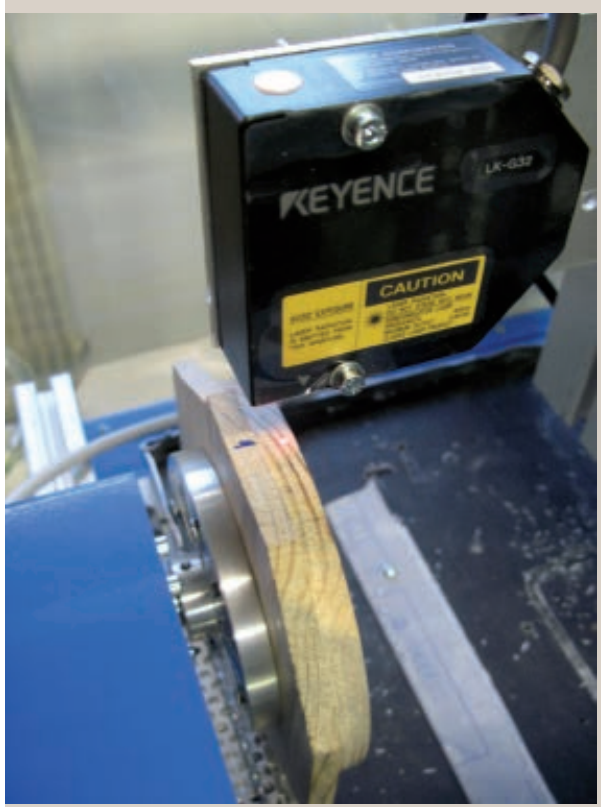

Photo 2 b.

Banc d'essai du test de la qualité de surface.

Zone de mesure par laser.

Photo H. Aknouche. 
Compte tenu du fait que l'arête de l'outil uti-

Efforts de coupe en fonction de l'angle du fil $\mathrm{CF}=$ contrefil $\mathrm{SF}=$ sens du fil

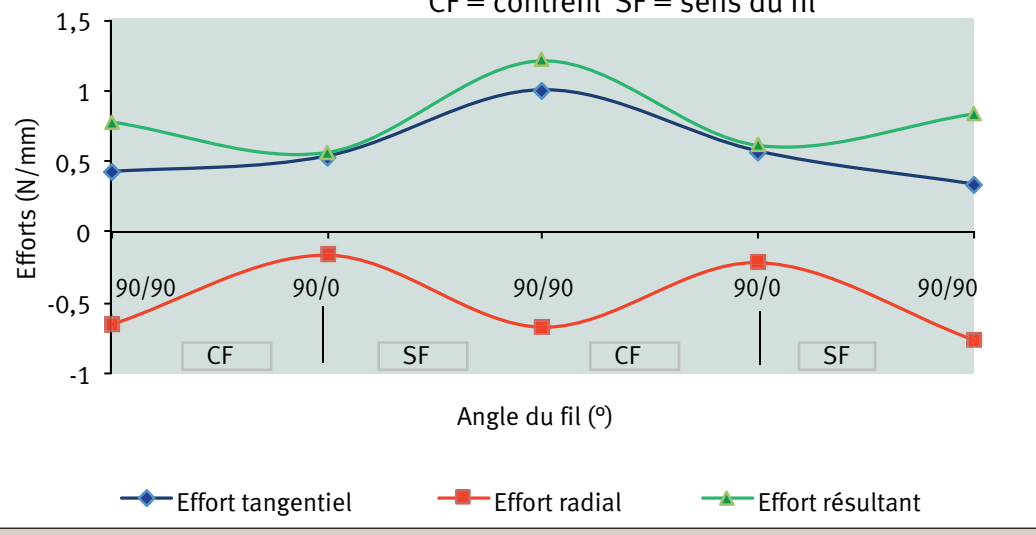

Figure 2.

Efforts de coupe en fonction de l'orientation du fil en usinant le pin d'Alep.

\section{Résultats et discussion}

\section{Évolutions des efforts de coupe}

La figure 2 confirme la forte influence de l'orientation du fil sur les efforts de coupe et fait apparaître que l'effort tangentiel est en valeur absolue environ deux fois plus important que l'effort radial. Il est remarquable aussi qu'au début de l'usinage les efforts de coupe sont plus importants du fait du mode de coupe $\left(90^{\circ} / 90^{\circ}\right)$, donc mettant en œuvre un cisaillement longitudinal/transversal. L'ensemble de ces observations a déjà été fait par Golı et al. (2009a, b), pour le défonçage du bois de pin Douglas, et Costes et al. (2004), pour l'usinage du bois d'érable.

Lorsque la position de l'arête de l'outil est à $0^{\circ}$ par rapport au fil (coupe en mode $90^{\circ} / 0^{\circ}$ ), les efforts sont réduits d'un facteur deux environ, ce qui est également un résultat communément admis.

Afin de situer le pin d'Alep parmi les autres bois, les efforts de coupe (ramenés à la longueur d'arête) obtenus ici ont été comparés avec ceux concernant le pin Douglas (tableau IV), un résineux mieux connu sur le plan de sa mise en œuvre (Golı et al., 2009b). Dans cette référence, la même méthode d'analyse du signal avait été utilisée mais pour une profondeur de passe de 1,5 mm. Pour la comparaison, les efforts de coupe spécifiques ont été utilisés, donc les valeurs ont été divisées par la surface moyenne du copeau. lisé pour usiner le pin d’Alep était en partie usée et que les conditions d'usinage n'étaient pas exactement les mêmes, on a constaté que les efforts de coupe spécifiques pour le pin d'Alep sont environ deux fois moins élevés que pour le pin Douglas.

\section{Résultats des photos numériques}

La planche 3 présente les photos numériques qui ont été recomposées. La photo 3a montre deux parties distinctes, la partie supérieure de l'usinage qui a été réalisée avec un outil neuf et l'autre avec un outil à arête tranchante émoussée. La bonne qualité d'usinage du pin d'Alep avec une arête neuve est bien visible, par contre celle usinée avec un outil usé présente quelques défauts. La photo $3 \mathrm{~b}$ montre la surface usinée du bois de pin Douglas. La qualité des surfaces générées en usinant le pin d’Alep (avec outil neuf ou émoussé) est bien meilleure que celle obtenue en usinant du pin Douglas.

\section{Résultats des essais de rugosité}

Les résultats issus des tests suivant les trois méthodes utilisées sont rassemblées dans la figure 3. On y voit les profils obtenus en faisant tourner le disque de $360^{\circ}$. La figure $3 a$ montre un profil non filtré obtenu en usinant du pin d'Alep avec un outil à arête émoussée, les grandes ondulations correspondant à des défauts d'excentricité du disque. Les figures $3 b, 3 c$, $3 d$ montrent les trois profils usinés du pin d'Alep avec un outil à arête émoussée, avec un outil avec une arête neuve, et du pin Douglas avec un outil neuf. En usinant du pin d'Alep avec un outil neuf et usé, on ne mesure pas de grandes différences. Il est remarquable que la qualité d'usinage du pin Douglas soit moindre que celle du pin d'Alep. Cette différence est confirmée par les index de rugosité en utilisant la valeur moyenne de l'écart-type du profil (figure 4).

La planche 4 présente les surfaces reconstruites à partir des 36 profils transversaux mesurés. La partie $4 a$ montre la surface usinée avec une arête neuve et la partie $4 \mathrm{~b}$ celle usinée avec une arête émoussée. Les différences de niveau se voient clairement entre les deux côtés, mais la qualité finale des deux surfaces est presque identique. La valeur de l'écart-type des profils 3D est de $0,084 \mathrm{~mm}$ pour le pin d'Alep et de 0,128 pour le pin Douglas, valeurs qui confirment la relation entre les valeurs des écarts-types du pin d'Alep et du pin Douglas mesurées dans les profils 2D.

\section{Tableau IV. \\ Comparaison entre les efforts de coupe mesurés sur le pin d'Alep et sur le pin Douglas par Goll et al. (2009b).}

\begin{tabular}{|c|c|c|c|c|}
\hline \multirow[t]{2}{*}{ Type d'usinage } & Douglas Fc (N/mm²) & Douglas Fn $\left(\mathrm{N} / \mathrm{mm}^{2}\right)$ & Pin d’Alep Fc (N/mm²) & Pin d'Alep Fn $\left(\mathrm{N} / \mathrm{mm}^{2}\right)$ \\
\hline & \multicolumn{2}{|c|}{ Efforts de coupe spécifiques } & \multicolumn{2}{|c|}{ Efforts de coupe spécifiques } \\
\hline $90^{\circ} / 0^{\circ}$ & 13,84 & 6,20 & 6,15 & 3,30 \\
\hline $90^{\circ} / 45^{\circ} \mathrm{SF}$ & 18,02 & 7,35 & 4,62 & 2,64 \\
\hline $90^{\circ} / 90^{\circ}$ & 17,73 & 6,77 & 4,22 & 3,86 \\
\hline $90^{\circ} / 45^{\circ} \mathrm{CF}$ & 13,55 & 5,77 & 5,18 & 2,34 \\
\hline
\end{tabular}


$\mathrm{a}$

b

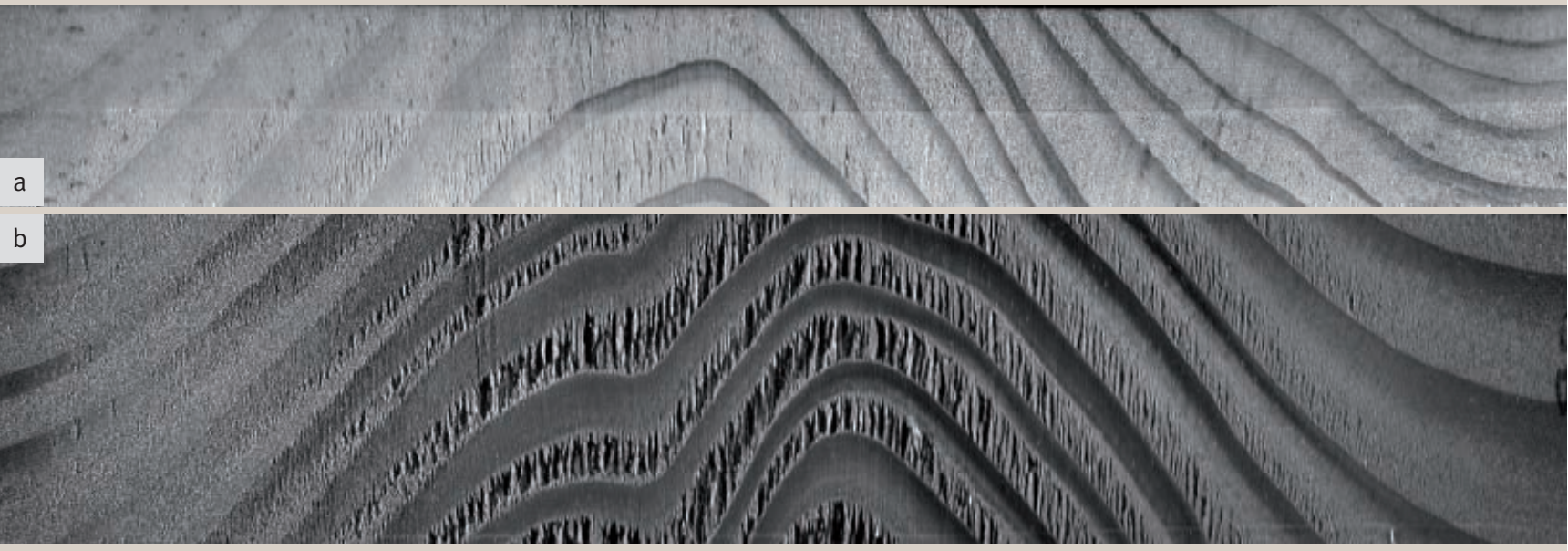

b

Photos 3.

Photos des surfaces usinées prises sur $180^{\circ}$ en tournant les roues et en faisant des photos

séquentielles avec une caméra digitale. a) Pin d’Alep, partie supérieure surface usinée avec

un outil à arête neuve, dans la partie inferieure surface usinée avec une arête émoussée.

b) Surface du pin Douglas.

Photos H. Aknouche.
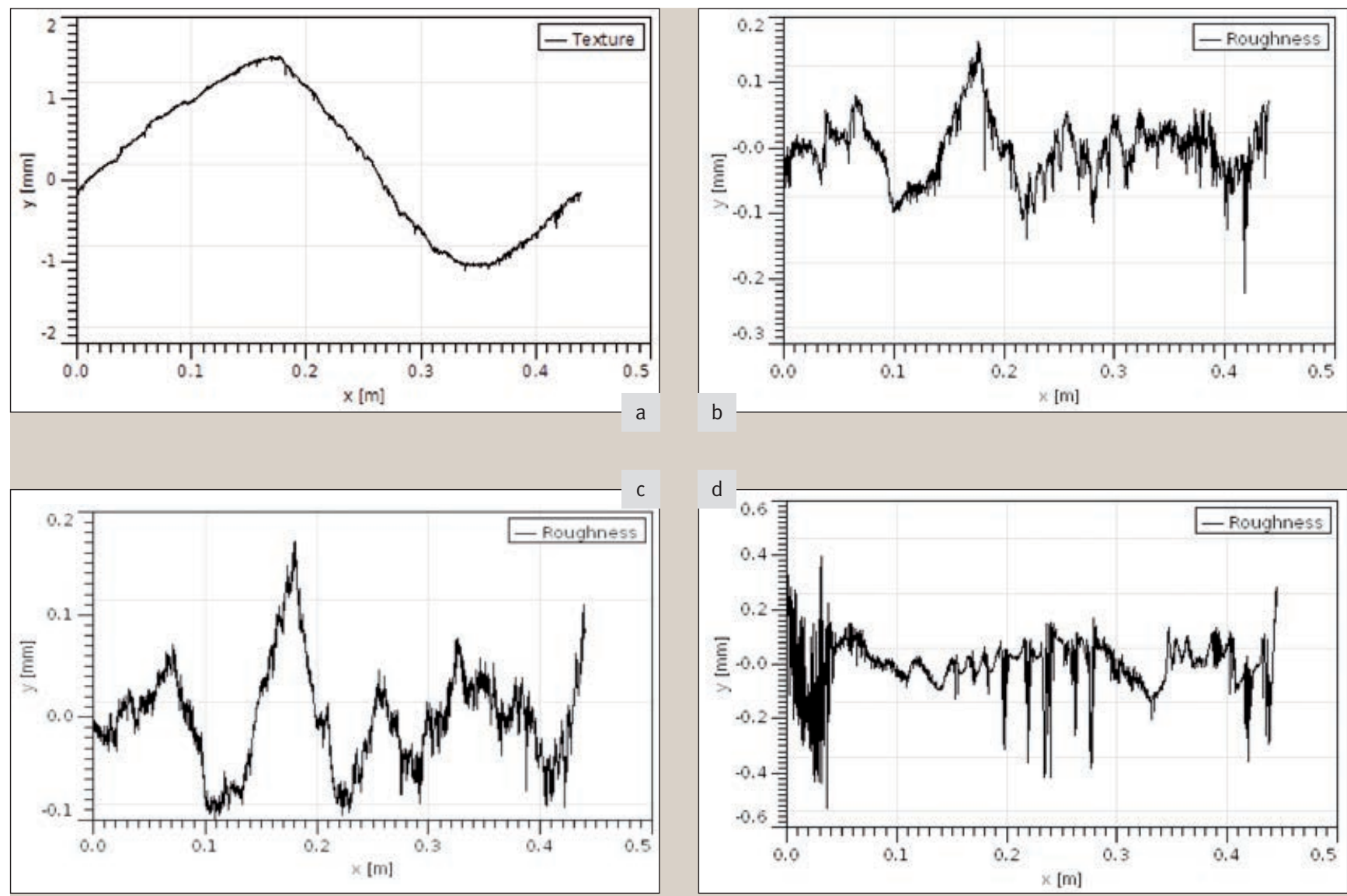

Figure 3.

Profils prélevés : a) usinage du pin d'Alep avec outil à arête émoussée sans filtrage de l'erreur de forme; b) usinage du pin d'Alep avec outil à arête émoussée après filtrage de l'erreur de forme ; c) usinage du pin d'Alep avec outil à arête neuve après filtrage de l'erreur de forme ; d) usinage du pin Douglas avec outil en carbure neuf après filtrage de l'erreur de forme. 
Profile RMS

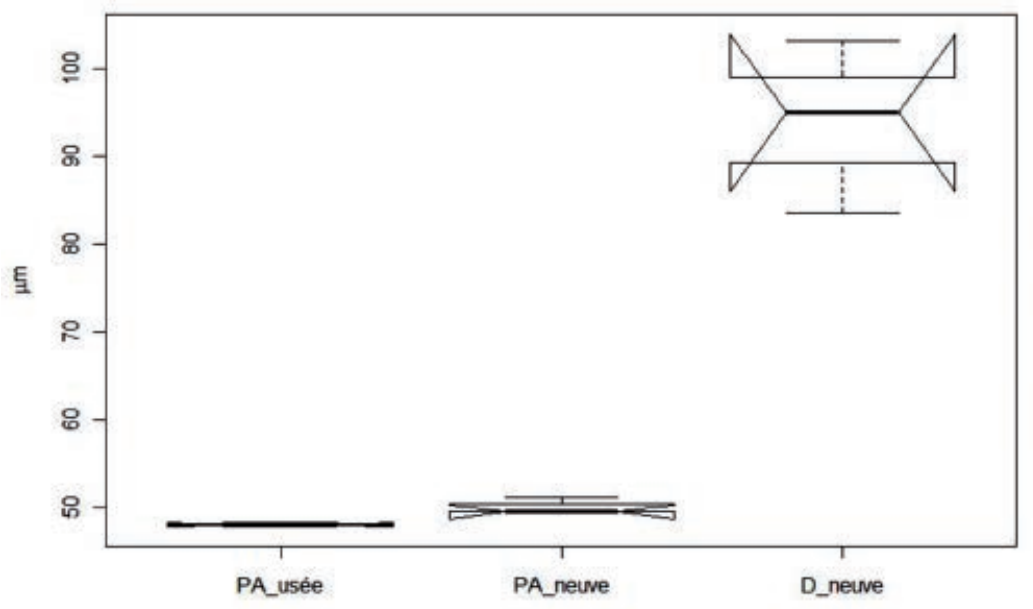

Figure 4.

Valeurs moyennes des écarts-types des profils longitudinaux mesurés sur du pin d'Alep usiné avec un outil à arête émoussée (PA usée), du pin d'Alep avec un outil à arête neuve (PA neuve) et du pin Douglas avec un outil neuf (D neuve).

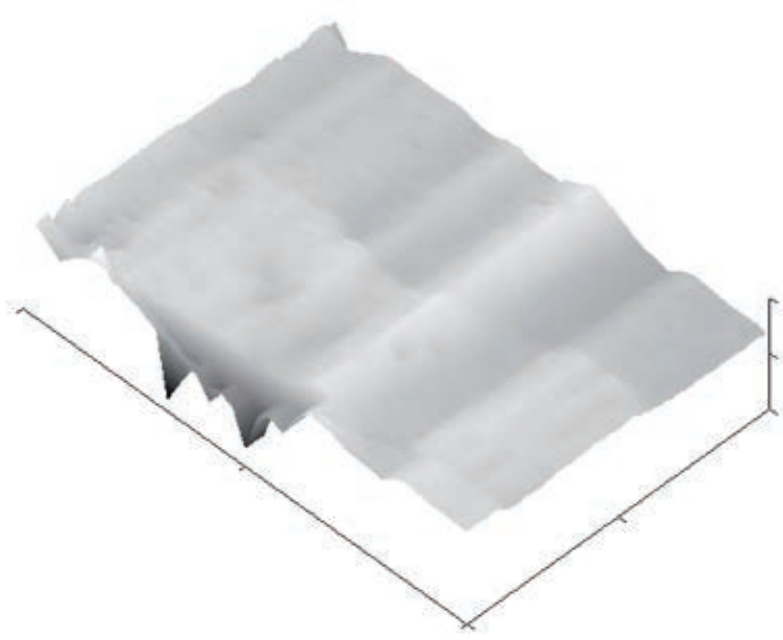

b

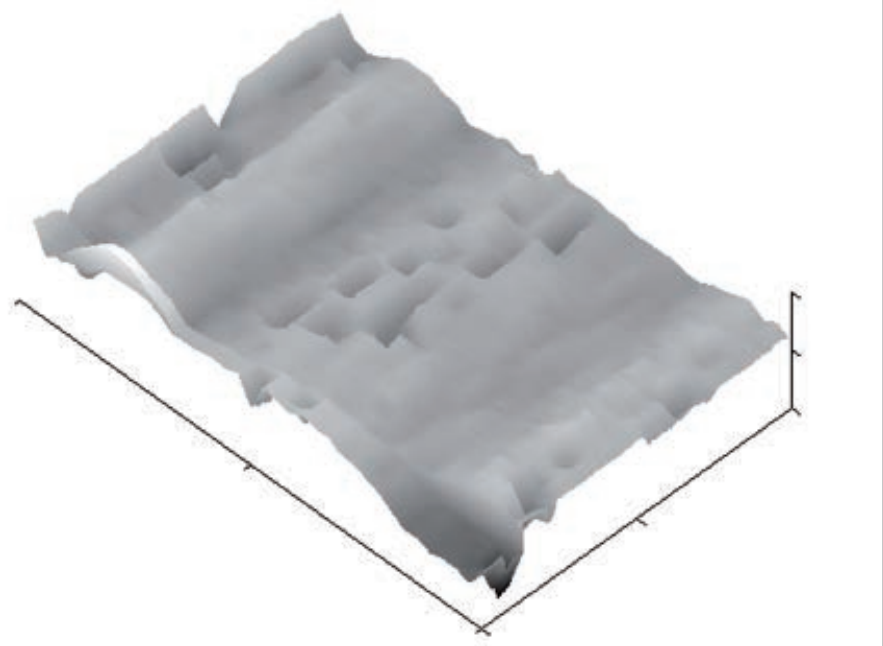

Photos 4.

Surface reconstruite par 36 profils mesurés transversalement. a) Pin d'Alep usiné avec un outil neuf et usé. b) Pin Douglas usiné avec un outil neuf. Photos H. Aknouche.

\section{Conclusion}

La procédure d'essai proposée dans cette étude s'est avérée très efficace pour une caractérisation simple d'une essence s'agissant des efforts de coupe nécessaires à sa transformation, et aussi par l'appréciation de la qualité des surfaces usinées. Elle a permis de comparer l'usinage avec une arête de coupe neuve ou émoussée du bois de pin d'Alep à celui du pin Douglas.

Les deux familles d'indicateurs peuvent êtres des facteurs déterminants dans l'industrie pour le choix des paramètres de coupe et du mode d'usinage des bois en général et du pin d'Alep en particulier. Les efforts de coupe pour usiner le pin d'Alep sont presque deux fois plus faibles que dans le cas du pin Douglas.

Les défauts superficiels des surfaces usinées sont facilement détectables par la méthode proposée, et des procédures d'intégration permettent la comparaison avec différentes méthodes existantes. La rugosité de la surface usinée du pin d'Alep est meilleure que celle du pin Douglas. En conclusion, le pin d'Alep est plus facile à usiner que le pin Douglas, que ce soit du point de vue des efforts de coupe ou de la qualité des surfaces obtenues.

\section{Remerciements}

Les auteurs remercient vivement les responsables du LaBoMaP Ensam ParisTech de Cluny pour avoir permis d'effectuer les différentes manipulations, du laboratoire d'Ivalsa/Cnr pour avoir permis de réaliser les mesures de la qualité des surfaces usinées, de l'Université M'Hamed Bougara-Boumerdès pour avoir financé la bourse de Hamid Aknouche. 


\section{Références bibliographiques}

AGUILLERA A., MARTIN P., 2001. Machining qualification of solid wood of Fagus silvatica L. and Picea excelsa L.: cutting forces, power requirements and surface roughness. Holz als Roh- und Werkstoff, 59 (6): 483-488.

AKNOUCHE H., OUTAHYON A., NOUVEAU C., MARCHAL R., ZERIZER A., BUTAUD J.-C., 2009. Tool wear effect on cutting forces: in routing process of Aleppo pine wood. Journal of Materials Processing Technology, 209: 2918-2922.

AKNOUCHE H., 2009. Étude de l'usure d'outils de coupe traités dans le domaine de l'usinage du bois. Thèse de doctorat, Université M'Hamed Bougara, Boumerdès, Algérie.

AXELSSON B. O. M., GRUNDBERG S. A., GRÖNLUND J. A., 1993. Tool wear when planning and milling. Measurement methodology and influencing factors. In: Proceedings of the 11th International Wood Machining Seminar, Norway, 25-27 May 1993. Oslo, Norvège, Norwegian Institute of Wood Technology, 159-176.

COSTES J.-P., PAK LIM KO, JI T., DECÈS-PETIT C., ALTINTAS Y., 2004. Orthogonal cutting mechanics of maple: modelling a solid wood-cutting process. Journal of Wood Science, 50 (1): 28-34.

CYRA G., TANAKA C., 2000. The effects of wood-fiber directions on acoustic emission in routing. Wood Science and Technology, 34 (3): 237-252.

GOLI G., LARRICQ P., MARCHAL R., NEGRI M., 2001. Surface quality: comparison among visual grading and $2 \mathrm{D}$ and $3 \mathrm{D}$ roughness measurements. In: Proceedings of the 15 th International Wood Machining Seminar. Los Angeles, CA, ÉtatsUnis, Loyola Marymount University, p. 459-471.

GOLI G., FIORAVANTI M., MARCHAL R., UZIELLI L., 2009a. Upmilling and down-milling wood with different grain orientations - theoretical background and general appearance of the chips. European Journal of Wood and Wood Products, 67 (3): 257-263.

GOLI G., FIORAVANTI M., MARCHAL R., UZIELLI L., BUSONI S., 2009b. Up-milling and down-milling wood with different grain orientations - the cutting forces behaviour. European Journal of Wood and Wood Products, 68 (4): 385-395.

HERNÁNDEZ R. E., ROJAS G., 2002. Effects of knife jointing and wear on the planed surface quality of sugar maple wood. Wood and Fiber Science, 34 (2): 293-305.

JIN W., CAI L., 1997. Study on the normal component force in oblique cutting of wood. Holz als Roh- und Werkstoff, 55: 118-120.

KOCH P., 1985. Utilization of hardwoods growing on southern pine sites. Volume II. Washington, DC, États-Unis, US Department of Agriculture, Forest Service, Agriculture Handbook $n^{\circ} 605$.
MARCHAL R., MOTHE F., DENAUD L. E., THIBAUT B., BLERON L., 2009. Cutting forces in wood machining - Basics and applications in industrial processes. A review COST Action E35 2004-2008: Wood machining - micromechanics and fracture. Holzforschung, 63 (2): 157-167.

MCKENZIE W., 1962. The relationship between the cutting properties of wood and its physical and mechanical properties. Forest Products Journal, 12 (6): 287-294.

NERI A. C., GONÇALVES R., HERNÁNDEZ R. E., 1999. Forças de corte ortogonal 90-0 em três espécies de madeira de eucalipto. Revista Brasileira de Engenharia Agrícola e Ambiental, 3 (2): 239-244.

NERI A. C., GONÇALVES R., HERNÁNDEZ R. E., 2000. Forças de corte ortogonal 90-90 em três espécies de madeira de eucalipto do estado de São Paulo. Revista Brasileira de Engenharia Agrícola e Ambiental, 4 (2): 275-280.

STEWART H. A., 1969. Effect of cutting direction with respect to grain angle on the quality of machined surface, tool force components, and cutting friction coefficient. Forest Products Journal, 19 (3): 43-46.

STEWART H. A., 1977. Optimum rake angle related to selected strength properties of wood. Forest Products Journal, 27 (1): 51-53.

STEWART H. A., 1980. Some surfacing defects and problems related to wood moisture content. Wood and Fiber, 12 (3): 175-182.

STEWART H. A., 1986. Fixed knife-pressure bar system for surfacing dry wood. Forest Products Journal, 36 (6): 52-56.

THIBAUT B., LOUP C., CHANSON B., DILEM A., 1992. La valorisation du pin d'Alep en zone méditerranéenne française. Forêt Méditerranéenne, 13 (3) : 226-233.

WOODSON G. E., KOCH P., 1970. Tool forces and chip formation in orthogonal cutting of loblolly pine. Washington, DC, États-Unis, US Department of Agriculture, Forest Service Research Paper SO-52, p. 356-362.

WYETH D. J., GOLI G., ATKINS A. G., 2009. Fracture toughness, chip types and the mechanics of cutting wood. A review COST Action E35 2004-2008: Wood machining - micromechanics and fracture. Holzforschung, 63 (2): 168-180. 